\title{
Measurement of the Spin and Magnetic Moment of ${ }^{31} \mathrm{Mg}$ : Evidence for a Strongly Deformed Intruder Ground State
}

\author{
G. Neyens, ${ }^{1}$ M. Kowalska, ${ }^{2}$ D. Yordanov, ${ }^{1}$ K. Blaum, ${ }^{3}$ P. Himpe, ${ }^{1}$ P. Lievens, ${ }^{4}$ S. Mallion,,${ }^{1}$ R. Neugart, ${ }^{2}$ N. Vermeulen, ${ }^{1}$ \\ Y. Utsuno, ${ }^{5}$ and T. Otsuka ${ }^{6}$ \\ ${ }^{1}$ Instituut voor Kern- en Stralingsfysica, Katholieke Universiteit Leuven, B-3001 Leuven, Belgium \\ ${ }^{2}$ Institut für Physik, Universität Mainz, D-55099 Mainz, Germany \\ ${ }^{3}$ CERN, Physics Department, CH-1211 Geneva 23, Switzerland \\ ${ }^{4}$ Laboratorium voor Vaste-Stoffysica en Magnetisme, Katholieke Universiteit Leuven, B-3001 Leuven, Belgium \\ ${ }^{5}$ Japan Atomic Energy Research Institute, Tokai, Ibaraki 319-1195, Japan \\ ${ }^{6}$ Department of Physics and Center for Nuclear Study, University of Tokyo, Hongo, Tokyo 113-0033, Japan \\ and RIKEN, Hirosawa, Wako-shi, Saitama 351-0198, Japan
}

(Received 20 October 2004; published 18 January 2005)

\begin{abstract}
Unambiguous values of the spin and magnetic moment of ${ }^{31} \mathrm{Mg}$ are obtained by combining the results of a hyperfine-structure measurement and a $\beta$-NMR measurement, both performed with an optically polarized ion beam. With a measured nuclear $g$ factor and spin $I=1 / 2$, the magnetic moment $\mu\left({ }^{31} \mathrm{Mg}\right)=-0.88355(15) \mu_{N}$ is deduced. A revised level scheme of ${ }^{31} \mathrm{Mg}(Z=12, N=19)$ with ground state spin/parity $I^{\pi}=1 / 2^{+}$is presented, revealing the coexistence of $1 \mathrm{p}-1 \mathrm{~h}$ and $2 \mathrm{p}-2 \mathrm{~h}$ intruder states below $500 \mathrm{keV}$. Advanced shell-model calculations and the Nilsson model suggest that the $I^{\pi}=1 / 2^{+}$ ground state is a strongly prolate deformed intruder state. This result plays a key role for the understanding of nuclear structure changes due to the disappearance of the $N=20$ shell gap in neutron-rich nuclei.
\end{abstract}

DOI: 10.1103/PhysRevLett.94.022501

Since Mayer and Jensen established the concept of shell structure in atomic nuclei, magic nucleon numbers have played a decisive role in describing the nuclear system [1]. About a quarter century later, the discovery of the anomalous ground state properties of ${ }^{31} \mathrm{Na}[2,3]$ suggested that the magic shell structure can be broken. Shell-model calculations allowing particle-hole ( $\mathrm{p}-\mathrm{h})$ excitations across the $N=20$ shell gap proposed that a group of nuclei with deformed ground states appears between $Z=10-12$ and $N=20-22$. Because the $\mathrm{p}-\mathrm{h}$ excited intruder states come lower in energy than the normal shell-model states, this region has been called the "island of inversion" [4]. In fact, $\beta$-decay experiments [5,6], intermediate energy Coulomb excitation [7], and in-beam $\gamma$-ray spectroscopy [8] confirmed the deformation of the even-even nuclei ${ }^{30} \mathrm{Ne},{ }^{32} \mathrm{Mg}$, and ${ }^{34} \mathrm{Mg}$. Moment measurements revealed that also the ground state of the $N=19$ nucleus ${ }^{30} \mathrm{Na}$ has an anomalous deformation [9] linked to this normalintruder inversion [10]. The following questions thus arise: Where is the boundary between the normal $s d$-shell nuclei and nuclei having an intruder ground state, and does it clearly exist at all? This general intriguing question about how widely nuclei with intruder ground states are spread and in what manner they become normal has not been answered, either experimentally or theoretically.

It has been suggested that the $N=20$ shell gap is changing from one nucleus to another [11,12] due to changes in the proton-neutron interaction. The boundary of the island of inversion can thus be shifted or smeared out, and intruder ground states might appear outside the earlier defined boundaries. Since the size of the shell gap is
PACS numbers: 21.10.-k, 23.20.-g, 27.30.+t, 32.10.Fn

related to the single-particle energies [determined mainly by the monopole part of the nucleon-nucleon $(N N)$ interaction], the mapping of the boundary is linked to one of the most basic and unanswered questions in present day nuclear structure physics: the microscopic mechanism to determine the monopole part of the $N N$ interaction.

We present in this Letter a measurement of the ground state spin and magnetic moment of the exotic even-odd nucleus ${ }^{31} \mathrm{Mg}(Z=12, N=19)$. The earlier observed anomalous lifetime and the branching intensities in its $\beta$ decay have never been explained [5,13], although the high level density suggested the presence of intruder states at low excitation energy [14]. However, unambiguous spin/ parity assignments are needed in order to establish the coexistence of normal $s d$-shell states with $1 \mathrm{p}-1 \mathrm{~h}$ and $2 \mathrm{p}$ $2 \mathrm{~h}$ intruder states. In addition to the ground state spin and parity, the magnetic moment value and sign provides direct information on the odd-neutron configuration.

The spin and magnetic moment of ${ }^{31} \mathrm{Mg}$ are measured by combining the results from two experimental techniques, based on the atomic hyperfine structure and on the nuclear interaction with external magnetic fields. Both methods rely on an optically polarized beam of ${ }^{31} \mathrm{Mg}^{+}$ions which are implanted into a crystal, where the angular asymmetry in the $\beta$ decay is detected. The experiments were performed at ISOLDE-CERN at the online collinear laser spectroscopy setup [15-17]. The ${ }^{31} \mathrm{Mg}$ beam was produced by bombarding a thick $\mathrm{UC}_{2}$ target with $1.4 \mathrm{GeV}$ protons $\left(1.2 \times 10^{13} / \mathrm{s}\right.$ on average) from the CERN PS-Booster. ${ }^{31} \mathrm{Mg}$ was selectively laser ionized with the resonance ionization laser ion source [18]. The ions 
were accelerated to $60 \mathrm{keV}$, and a mass separated ${ }^{31} \mathrm{Mg}^{+}$ beam with a typical intensity of $1.5 \times 10^{5}$ ions/s (and less than $5 \%$ contamination from surface-ionized ${ }^{31} \mathrm{Na}$ ) was guided to the collinear laser spectroscopy setup. The $\beta$-decay half-life was measured to be in agreement with the earlier published value of 250(30) ms [5].

The $\mathrm{Mg}^{+}$ions are polarized through optical Zeeman pumping with a circularly polarized laser beam propagating along the ion beam. The polarization axis parallel to the laser beam is maintained by a weak magnetic guiding field over the interaction zone. Via the hyperfine interaction and adiabatic decoupling of the electron and nuclear spins, the resonantly induced electron polarization is partly transferred to the nucleus. The laser wavelength was set to the Doppler-shifted resonance value for the $D_{2}$ line $\left(3 s^{2} S_{1 / 2} \leftrightarrow 3 p^{2} P_{3 / 2} ; 35760.88 \mathrm{~cm}^{-1}\right)$. About $15 \mathrm{~mW}$ of UV power $(\lambda \approx 280 \mathrm{~nm})$ was obtained from an externalcavity frequency doubling ring, coupled to the output of a cw dye laser (Pyrromethene 556) which was pumped by an $\mathrm{Ar}^{+}$laser. By varying the velocity of the ions, applying a tunable high voltage to the interaction zone, the hyperfine structure was scanned. The optically induced resonances are observed through the asymmetry in the $\beta$ decay of the polarized ${ }^{31} \mathrm{Mg}$ nuclei, after implantation into a $\mathrm{MgO}$ single crystal placed in a transverse magnetic field $B$. The coincidence counts from two $\beta$ telescopes (each consisting of two thin plastic scintillators), placed at $0^{\circ}$ and $180^{\circ}$ with respect to the magnetic field, allowed the normalized $\beta$-decay asymmetry $\left[N\left(0^{\circ}\right)-N\left(180^{\circ}\right)\right] /$ $\left[N\left(0^{\circ}\right)+N\left(180^{\circ}\right)\right]$ to be deduced.

In Figs. 1(a) and 1(b) the $\beta$-decay asymmetry is shown as a function of the scanned acceleration voltage, for lefthanded $\left(\sigma^{-}\right)$and right-handed $\left(\sigma^{+}\right)$polarized laser light. Apart from the sign changes in the resonance amplitudes, which depend on details of the optical pumping and decoupling process as explained in [9], the line positions are easily understood as arising from the different hyperfinestructure components $F$ of the atomic ground and excited states [Fig. 1(c)]. For example, the splitting between the electronic $J=1 / 2$ ground state levels equals $\Delta \nu(x)=$ $A(x)[I(x)+1 / 2]$ and depends on the nuclear spin $I$. The hyperfine constant $A(x)$ depends on the nuclear $g$ factor and the isotope independent magnetic hyperfine field $H_{e}$ [15], $A(x)=g(x) \mu_{N} H_{e} / J$. To simulate the line positions, the hyperfine fields induced by the ${ }^{2} S_{1 / 2}$ and ${ }^{2} P_{3 / 2}$ electron states need to be known. These have been deduced from hyperfine-structure data on the stable isotope ${ }^{25} \mathrm{Mg}$, reported from an optical pumping laser-rf double resonance experiment in a Penning trap [19]. The essential number is the $3 s^{2} S_{1 / 2}$ magnetic hyperfine-structure constant $A_{\text {g.s. }}\left({ }^{25} \mathrm{Mg} \mathrm{Mg}\right)=-596.254376(54) \mathrm{MHz}$. Knowing the spin $I=5 / 2$ and magnetic moment $\mu=$ $-0.85545(8) \mu_{N}$ of ${ }^{25} \mathrm{Mg}$ [20], the hyperfine constants for ${ }^{31} \mathrm{Mg}$ can be deduced with the nuclear $g$ factor as a parameter. Assuming the measured $g$ factor (as described

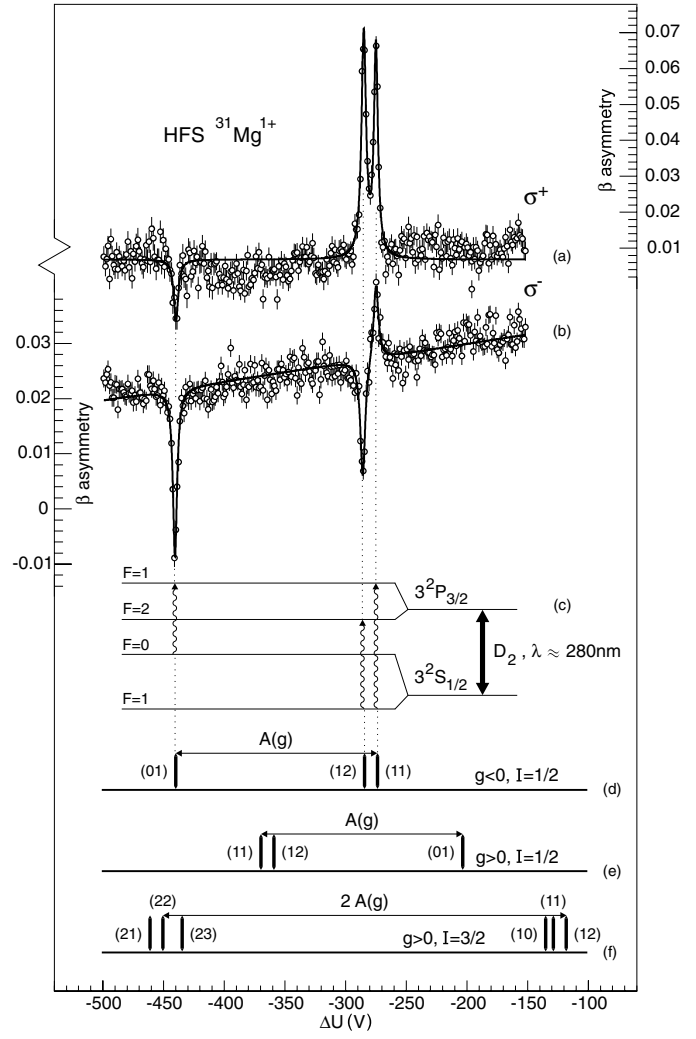

FIG. 1. (a),(b) Hyperfine structure (HFS) spectra of $\sigma^{+} / \sigma^{-}$ optically polarized ${ }^{31} \mathrm{Mg}^{+}$ions, observed via the asymmetry in the nuclear $\beta$ decay after implantation into $\mathrm{MgO}$. (c) Hyperfine structure in the transition $3 s^{2} S_{1 / 2} \rightarrow 3 p^{2} P_{3 / 2}\left(D_{2}\right.$ line) assuming a nuclear spin $I=1 / 2$. (d)-(f) Simulated spectra assuming $I=1 / 2$ or $I=3 / 2$, using the absolute $g$ factor measured by NMR. The allowed transitions are labeled by the total angular momenta $F$ of the ground- and excited-state hyperfine levels.

below) with a negative or a positive sign, this results in $A_{\text {g.s. }}\left({ }^{31} \mathrm{Mg}\right)= \pm 3079.4(8) \mathrm{MHz}$. For a nuclear spin $I=$ $1 / 2$ this gives the line positions as shown in Figs. 1(d) and 1(e). The good agreement of Fig. 1(d) with the observed line splitting clearly decides for the ground state spin $I=1 / 2$ and a negative $g$ factor. Any other spin assumption would involve a much larger splitting, as shown in Fig. 1(f) for the example of $I=3 / 2$. The spin $I=1 / 2$ is also manifest in the feature that only three resonances are detected in the hyperfine-structure scans. Now the adopted nuclear $g$ factor remains to be confirmed by a nuclear magnetic resonance (NMR) measurement.

Maximum polarization of the ${ }^{31} \mathrm{Mg}$ beam is obtained for $\sigma^{+}$light, with the Doppler-tuning voltage fixed to the resonance value of the (12) transition defined in Fig. 1(d). This is used for a NMR measurement on ${ }^{31} \mathrm{Mg}$ implanted into $\mathrm{MgO}$. Resonant destruction of the nuclear polarization is induced by a rf magnetic field at the Larmor frequency $\nu_{L}=g \mu_{N} B / h$. The resonance is recorded by measuring the $\beta$ asymmetry as a function of the applied $\mathrm{rf}$ frequency, as shown in Fig. 2. In total, ten measurements 


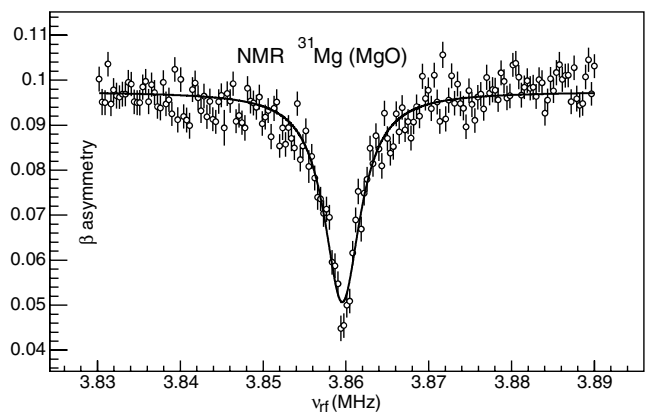

FIG. 2. Typical $\beta$ NMR for ${ }^{31} \mathrm{Mg}$ implanted in $\mathrm{MgO}$.

have been performed for different rf powers, and a statistical average over the resonance positions was taken to obtain the Larmor frequency $\nu_{L}\left({ }^{31} \mathrm{Mg}\right)=$ $3859.73(18) \mathrm{kHz}$. To calibrate the magnetic field, we measured the NMR spectrum for a polarized ${ }^{8} \mathrm{Li}$ beam implanted in the same $\mathrm{MgO}$ crystal: $\nu_{L}\left({ }^{8} \mathrm{Li}\right)=$ $1807.03(2) \mathrm{kHz}$. From both Larmor frequencies, and using $g\left({ }^{8} \mathrm{Li}\right)=0.826780(9)$ [20], the absolute value of the ${ }^{31} \mathrm{Mg}$ $g$ factor is deduced. This value needs to be corrected for diamagnetic shielding, using the numbers from Raghavan's table [20], resulting in $\left|g\left({ }^{31} \mathrm{Mg}\right)\right|=$ 1.7671(3). The error accounts for a possible $5 \times 10^{-5}$ magnetic field drift over the $48 \mathrm{~h}$ period between the measurements and a similar uncertainty due to a possible difference in the beam positions on the crystal. This value, with a negative sign, gives the hyperfine-structure simulation of Fig. 1(d), confirming not only that the ground state spin of ${ }^{31} \mathrm{Mg}$ is $I=1 / 2$ but also that the magnetic moment is negative, namely, $\mu\left({ }^{31} \mathrm{Mg}\right)=-0.88355(15) \mu_{N}$. The sign and magnetic moment value confirm the positive parity assigned to this $I=1 / 2$ level (see further).

Earlier $\beta$-decay experiments already assigned a positive parity to the ground state and most of the low-lying states, based on the fact that they are fed via the $\beta$ decay from the positive parity ${ }^{31} \mathrm{Na}$ ground state [14]. One level at $461 \mathrm{keV}$ was observed only via the $\beta$-delayed neutron decay of ${ }^{32} \mathrm{Na}(N=21)$ and therefore assigned a negative parity. No firm spin assignments could be made. Now, with our ground state spin/parity $I^{\pi}\left({ }^{31} \mathrm{Mg}\right)=1 / 2^{+}$, we can make tentative spin/parity assignments to the first excited states of ${ }^{31} \mathrm{Mg}$ (Fig. 3) using the multipolarity assignments made by Klotz et al. [14]. The negative parity level at $461 \mathrm{keV}$ is most likely the $I^{\pi}=7 / 2^{-}(1 \mathrm{p}-1 \mathrm{~h})$ intruder state, confirmed recently from a lifetime measurement on the $240 \mathrm{keV} \gamma$ decay [21]. Further, the earlier observed hindered $\beta$ decay to the ground state of ${ }^{31} \mathrm{Al}$ [14], known to have $I^{\pi}=5 / 2^{+}$[22], can also be understood. A new measurement of the ${ }^{31} \mathrm{Mg} \beta$ decay has revealed an even weaker ground state feeding [23], as expected for a second forbidden $1 / 2^{+}$to $5 / 2^{+}$transition.

The level scheme is compared to recent shell-model calculations in Fig. 3. First, we compare to a calculation performed with the USD interaction, where protons and neutrons are in the positive parity $s d$ shell only [13]. The lowest $1 / 2^{+}$state is in this case predicted around $2.5 \mathrm{MeV}$ above the normal $3 / 2^{+}$ground state (labeled $0 \mathrm{p}-0 \mathrm{~h}$ in Fig. 3). The complete disagreement with the observed high level density below $500 \mathrm{keV}$, including both positive and negative parity states, suggests the need to include neutron excitations into the negative parity $p f$ shell [14]. Such calculations in the $s d-p f$ model space have been performed using two approaches.

With the ANTOINE shell-model code [12], using the interaction described in [24], calculations are performed in the full $s d-p f$ space. Intruder states are calculated by blocking, respectively, one or two neutrons in the $p f$ shell (1p-1h, 2p-2h in Fig. 3), resulting in low-lying positive and negative parity states below $1 \mathrm{MeV}$. While the $1 \mathrm{p}-1 \mathrm{~h}$ intruder states are predicted to occur well below the $2 \mathrm{p}$ $2 \mathrm{~h}$ states (contrary to the experimental situation), these calculations do predict the correct ordering within each doublet $\left(1 / 2^{+}\right.$below $3 / 2^{+}$, and $3 / 2^{-}$below $\left.7 / 2^{-}\right)$. The calculated magnetic moment (using free-nucleon $g$ factors) of the pure $2 \mathrm{p}-2 \mathrm{~h} 1 / 2^{+}$intruder state is in good agreement with the observed value $\left(\mu=-0.84 \mu_{N}\right.$ compared to $\left.\mu_{\text {exp }}=-0.88355 \mu_{N}\right)$, suggesting that the wave function of the observed ground state is close to a pure $2 \mathrm{p}-2 \mathrm{~h}$ intruder. The lowest $1 / 2^{-}$state lies around $1.5 \mathrm{MeV}$ and has a positive magnetic moment $\mu=+1.54 \mu_{N}$.

Another set of calculations, including mixing between normal and intruder $n \mathrm{p}-n \mathrm{~h}$ configurations, has been performed with the Monte Carlo shell-model (MCSM) [25] in the $s d-p_{3 / 2} f_{7 / 2}$ space ("mixed $n \mathrm{p}-n \mathrm{~h}$ " in Fig. 3). The interaction for this model space is described in [26] and was used to describe a variety of nuclei, many of which have intruder dominant low-lying states [10,26,27]. While these mixed calculations reproduce better the high level density below $500 \mathrm{keV}$, they also cannot reproduce the

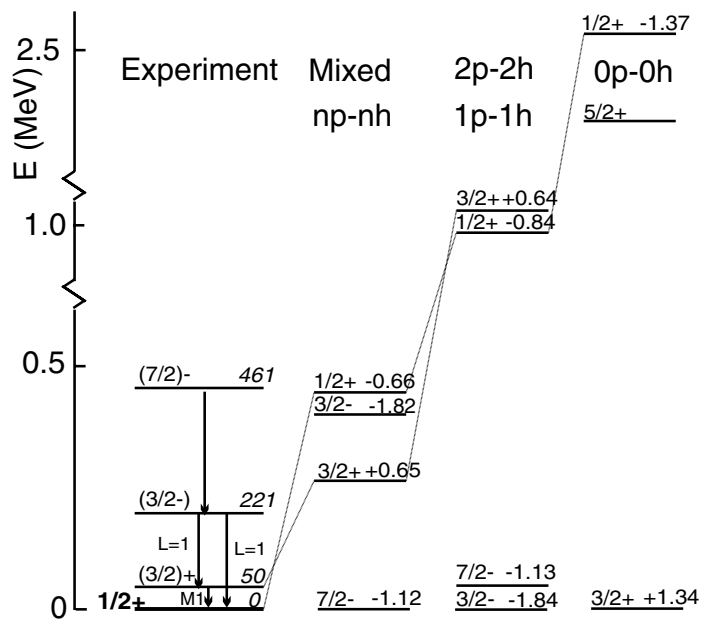

FIG. 3. Partial experimental level scheme of ${ }^{31} \mathrm{Mg}$, with new spin/parity assignments, compared to various shell-model calculations (see text for details). The magnetic moments of theoretical levels are mentioned on the right (units $\mu_{N}$ ). 
correct ordering of the intruder levels. The magnetic moment for the $1 / 2^{+}$level $\left(\mu=-0.66 \mu_{N}\right)$, dominated by more than $90 \%$ of $2 \mathrm{p}-2 \mathrm{~h}$ intruder configurations, shows a less good agreement with experiment than for the pure $2 \mathrm{p}$ $2 \mathrm{~h}$ state calculated with the interaction from [24]. This shows the sensitivity of the magnetic moment to small changes in the configuration, due to differences in the model space and single-particle energies.

The fact that the pure intruder calculations with the interaction from Ref. [24] predict the correct ordering within each doublet and reproduce better the magnetic moment than the calculations with the interaction from [26] may guide us to improve the interactions, particularly in their monopole and/or spin-isospin parts. The spacing between the $1 \mathrm{p}-1 \mathrm{~h}$ and $2 \mathrm{p}-2 \mathrm{~h}$ states is closely related to the $N=20 s d-p f$ shell gap. The reduction of this gap from ${ }^{40} \mathrm{Ca}$ down to ${ }^{32} \mathrm{Mg}$ is different for the $s d-p f$ interaction (from 7.5 to $5.3 \mathrm{MeV}$ ) and the $s d-p_{3 / 2} f_{7 / 2}$ interaction (from 6 to $4 \mathrm{MeV}$ ). The better agreement of the MCSM calculations with the high experimental level density is an indication that indeed the shell gap is strongly reduced in the $\mathrm{Mg}$ isotopes. Such a reduced gap creates extensive particle-hole excitations from the $s d$ shell to the $p f$ shell, making ${ }^{31} \mathrm{Mg}$ rather soft against deformation and inducing a near degeneracy of $1 \mathrm{p}-1 \mathrm{~h}$ and $2 \mathrm{p}-2 \mathrm{~h}$ states. In fact, in a Nilsson model picture, the [200]1/2+ Nilsson orbit is occupied by the last neutron of ${ }^{31} \mathrm{Mg}$ for a large prolate deformation $(\beta \sim+0.4)$. Thus, the present firm spin value suggests a strong prolate deformation of the ${ }^{31} \mathrm{Mg}$ ground state. This is confirmed by the shell-model calculations, where the quadrupole moment of the mixed $n \mathrm{p}-n \mathrm{~h}$ and the pure $2 \mathrm{p}-2 \mathrm{~h}$ states are calculated to be respectively $Q\left(3 / 2^{+}\right)=-13.6$ and $-14.6 e \mathrm{fm}^{2}$ and with transition moments $B\left(E 2 ; 3 / 2^{+} \rightarrow 1 / 2^{+}\right)=85$ and $87 e^{2} \mathrm{fm}^{4}$. These $E 2$ quantities are consistent with a prolate deformed $K=1 / 2$ band having an intrinsic quadrupole moment $Q_{0} \sim 65 \mathrm{fm}^{2}$ and deformation $\beta \sim+0.44$.

In conclusion, we determined the spin/parity of the ${ }^{31} \mathrm{Mg}$ ground state as $I^{\pi}=1 / 2^{+}$and present an updated level scheme with tentative spin/parity assignments to the lowest excited states. Calculations using the most recent shellmodel interactions for the $s d-p f$ shell cannot reproduce the experimentally observed level ordering, but they predict the observed coexistence of $1 \mathrm{p}-1 \mathrm{~h}$ and $2 \mathrm{p}-2 \mathrm{~h}$ intruder configurations at low excitation energy. A comparison of the experimental magnetic moment with the calculations for pure intruder and mixed wave functions suggests that the observed $1 / 2^{+}$ground state is a nearly pure $2 \mathrm{p}-2 \mathrm{~h}$ intruder state, which in a Nilsson model approach is related to the strongly deformed $[200] 1 / 2^{+}$level.

The spin/parity assignments made in this work, together with the static moments of this and other odd- $A$ nuclei, are thus providing a key element for further investigating the shell gap evolution in this region of the nuclear chart.

This work is part of the Ph.D. theses of M. K. and D. Y. We are very grateful to F. Nowacki, A. Poves, and N. A. Smirnova for help with the ANTOINE calculations and fruitful discussions about the results. This work has been supported by the German Ministry for Education and Research (BMBF) under Contract No. 06MZ175, by the IUAP Project No. p5-07 of OSCT Belgium, and by the FWO-Vlaanderen, by Grant-in-Aid for Specially Promoted Research (13002001) and for Young Scientists (14740176) from the MEXT of Japan. P.H. acknowledges financial support from IWT Flanders. The authors thank the ISOLDE technical group for their assistance.

[1] M. G. Mayer and J.H. D. Jensen, Elementary Theory of Nuclear Shell Structure (John Wiley and Sons, London, 1955).

[2] C. Thibault et al., Phys. Rev. C 12, 644 (1975).

[3] G. Huber et al., Phys. Rev. C 18, 2342 (1978).

[4] E. K. Warburton, J. A. Becker, and B.A. Brown, Phys. Rev. C 41, 1147 (1990).

[5] C. Detraz et al., Phys. Rev. C 19, 164 (1979).

[6] D. Guillemaud-Mueller et al., Nucl. Phys. A426, 37 (1984).

[7] T. Motobayashi et al., Phys. Lett. B 346, 9 (1995).

[8] B. V. Pritychenko et al., Phys. Rev. C 63, 011305(R) (2000).

[9] M. Keim et al., Eur. Phys. J. A 8, 31 (2000).

[10] Y. Utsuno et al., Phys. Rev. C 70, 044307 (2004).

[11] T. Otsuka et al., Phys. Rev. Lett. 87, 082502 (2001).

[12] E. Caurier, F. Nowacki, A. Poves, and J. Retamosa, Phys. Rev. C 58, 2033 (1998).

[13] B. H. Wildenthal, M.S. Curtin, and B. A. Brown, Phys. Rev. C 28, 1343 (1983).

[14] G. Klotz et al., Phys. Rev. C 47, 2502 (1993).

[15] R. Neugart, Nucl. Instrum. Methods Phys. Res. 186, 165 (1981).

[16] E. Arnold et al., Phys. Lett. B 197, 311 (1987).

[17] W. Geithner et al., Phys. Rev. Lett. 83, 3792 (1999).

[18] U. Köster et al., Nucl. Instrum. Methods Phys. Res., Sect. B 204, 347 (2003).

[19] W. M. Itano and D. J. Wineland, Phys. Rev. A 24, 1364 (1981).

[20] P. Raghavan, At. Data Nucl. Data Tables 42, 189 (1989).

[21] H. Mach (private communication).

[22] D. Borremans et al., Phys. Lett. B 537, 45 (2002).

[23] F. Marechal (private communication).

[24] S. Nummela et al., Phys. Rev. C 63, 044316 (2001).

[25] T. Otsuka et al., Prog. Part. Nucl. Phys. 47, 319 (2001).

[26] Y. Utsuno, T. Otsuka, T. Mizusaki, and M. Honma, Phys. Rev. C 64, 011301(R) (2001).

[27] Y. Utsuno, T. Otsuka, T. Mizusaki, and M. Honma, Phys. Rev. C 60, 054315 (1999). 